\title{
Right jugular foramen dural arteriovenous fistula Cognard IV
}

\author{
Ivo Peto, MD, Hussam Abou AI-Shaar, MD, and Amir R. Dehdashti, MD, FACS
}

Department of Neurosurgery, North Shore University Hospital, Manhasset, New York

Posterior fossa dural arteriovenous fistulas (dAVFs) are rare vascular malformations. They carry a significant risk of hemorrhage if associated with cortical venous reflux. A 70-year-old man presented with right-sided medullary hemorrhage with pronounced Wallenberg syndrome. Angiography demonstrated right jugular foramen dAVF with direct brainstem venous reflux (Cognard IV). It was fed from multiple branches of the external carotid artery and the vertebral artery, and draining into the ascending pontomesencephalic vein. Primary two-stage transarterial embolization was performed with near-total occlusion of the fistula to prevent it from rebleeding in the acute phase. Because of the patient's significant neurological deficit, the surgery was deferred to later and if the DAVF showed further progression. Follow-up angiography 8 months later demonstrated obvious recurrence and progression of the fistula from adjacent feeders. In the meantime, the patient had a remarkable recovery from the Wallenberg symptoms. To achieve complete occlusion of the fistula, a right far lateral approach was chosen with complete disconnection of the fistula. Postoperative angiography confirmed complete occlusion of the fistula, and the patient remained intact from the procedure.

The video can be found here: https://youtu.be/DJvpa8G4olc.

KEYWORDS dural arteriovenous fistula; surgical disconnection; arteriovenous malformation; cortical venous reflux; video 